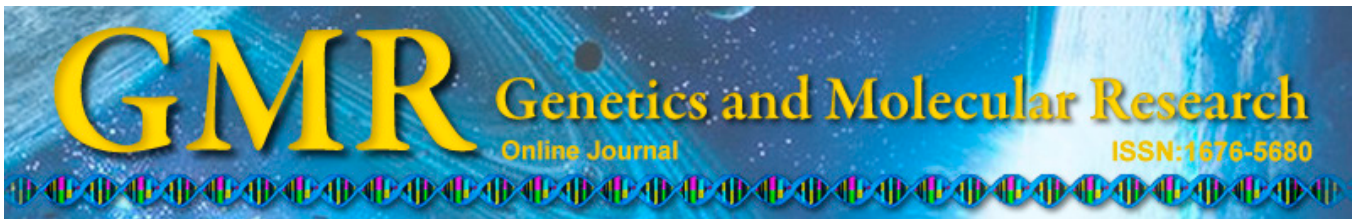

\title{
Genetic diversity of wild soybean populations in Dongying, China, by simple sequence repeat analysis
}

\author{
Y.H. Wang*, X.J. Zhang* and S.J. Fan \\ Key Laboratory of Plant Stress Research, College of Life Science, \\ Shandong Normal University, Jinan, China \\ *These authors contributed equally to this study. \\ Corresponding author: S.J. Fan \\ E-mail: fanshoujin007@163.com
}

Genet. Mol. Res. 14 (3): 11613-11623 (2015)

Received February 4, 2015

Accepted May 25, 2015

Published September 28, 2015

DOI http://dx.doi.org/10.4238/2015.September.28.13

\begin{abstract}
Annual wild soybean (Glycine soja Sieb. et Zucc.), the ancestor of cultivated soybean (G. max), is believed to be a potential gene source for further improvement of soybean to cope with environmental stress. In this study, 10 simple sequence repeat (SSR) markers were used to evaluate the genetic diversity and population genetic structure in five wild soybean populations using 195 accessions collected from Dongying, China. Ten SSR markers yielded 90 bands, with an average of nine bands per marker. The percentage of polymorphic loci $(\mathrm{P})$ was $97.78 \%$, the distribution of expected heterozygosity $\left(H_{\mathrm{E}}\right)$ was $0.1994-$ 0.4460 with an average of 0.3262 , and the distribution from Shannon's information index $(I)$ was $0.3595-0.6506$ with an average of 0.5386 . The results showed that wild soybean had a high degree of genetic diversity at the species level. Nei's differentiation coefficient $\left(F_{\mathrm{ST}}\right)$ was 0.1533 , and gene flow $\left(N_{\mathrm{m}}\right)$ was 1.3805 , which indicated that genetic variation mainly existed within populations and that there was a certain level of gene exchange between populations. Some genetic differentiation occurred among populations, although this was not significant. Cluster
\end{abstract}


analysis indicated that there was no significant correlation between the genetic structure of wild soybean populations and their geographic distribution, and the clustering results may be relatively consistent with the habitats of the accessions. In the present study, the genetic diversity of wild soybeans showed a broad genetic base and enables suggestions for the conservation of this plant to be made.

Key words: Wild soybean; Simple sequence repeat; Genetic diversity; Conservation

\section{INTRODUCTION}

Wild soybean (Glycine soja) is an annual twining herb that belongs to the genus Glycine, in the family Leguminosae, and is distributed throughout East Asia, including China, Korea, Japan, and the far eastern regions of Russia. In China, it is distributed in every province except Xinjiang, Qinghai, and Hainan. Wild soybeans, the ancestors of cultivated soybeans (G. max), are important sources of major genes that offer plant resistance to pests, diseases, and environmental stresses. The study of their genetic diversity is invaluable for the efficient utilization, conservation, and management of germplasm collections.

Most of the previous studies on G. soja were based on morphological traits and enzymes (Abe et al., 1992; Li et al., 1995; Gai et al., 2000; Wu et al., 2004). Due to the influence of a changing environment on morphological evaluation and the limited information provided by enzyme analysis, the focus of current research on the genetic diversity of wild soybean has transitioned from morphology and enzymes to investigations at the molecular level. Kuroda et al. (2006) analyzed 77 natural wild soybean populations from Japan, using 20 microsatellite loci in 616 individual plants and concluded that wild soybeans had undergone introgression from cultivated soybeans. Zhou et al. (2002) studied 16 samples of wild soybeans that originated from a region at $25^{\circ} \mathrm{N}$ latitude using randomly amplified polymorphic DNA (RAPD) and simple sequence repeat (SSR) markers. They indicated that there is a vast amount of genetic variation within the population. Wang et al. (2010) analyzed 370 accessions of $G$. soja that were collected from the eastern area of Hebei Province and two accessions from Heilongjiang Province in China using SSR markers. They found that obvious genetic differences existed between the materials collected near the coastline and the materials collected in the inland region. Zhao et al. (2006) analyzed the genetic diversity of the wild soybean population in the Shanghai Jiangwan district in China using three molecular marker technologies (AFLP, ISSR, and SSR) and compared different genetic diversity indices using computer simulation. They found that the genetic diversity indices within the same wild soybean population were considerably different when estimated by different molecular marker techniques, and that $\mathrm{P}$ values were relatively more reliable for comparing genetic diversity. This study further showed that 30-40 individuals could capture over $90 \%$ of the total genetic diversity of the wild soybean population when using the $\mathrm{P}$ value as the determining factor. Their study provided a scientific basis for the assessment of genetic diversity and appropriate sampling strategies for $G$. soja.

Genetic diversity studies on wild soybean have been recognized by some researchers, but none of those studies reported the genetic diversity of wild soybean populations in the 
Dongying region in China. In 2004, China established 1000 acres of wild soybean as a natural reserve in the city of Dongying, in Shandong Province to better protect these precious wild plant resources. In order to utilize, conserve, and manage wild soybean effectively in the Dongying region, we analyzed the genetic diversity, genetic variation, and genetic structure among and within these populations using SSR markers.

\section{MATERIAL AND METHODS}

\section{Plant materials}

A total of 195 wild accessions were used in this study, which were collected from five natural populations in Dongying (Table 1 and Figure 1). In order to better assess the genetic diversity of wild soybeans, the wild soybean seeds were collected according to the findings of Zhao et al. (2006). From each population, 30-50 individuals were collected by GPS positioning, and the space between adjacent individuals was $5 \mathrm{~m}$.

\begin{tabular}{|c|c|c|c|c|}
\hline Production area & Code & Accession No. & Latitude of the site $\left({ }^{\circ} \mathrm{N}\right)$ & Longitude of the site $\left({ }^{\circ} \mathrm{E}\right)$ \\
\hline Kenli County & KL & 50 & $\mathrm{~N} 37^{\circ} 44^{\prime} 44.440^{\prime \prime}$ & E11909'12.176" \\
\hline Hekou District & $\mathrm{HK}$ & 43 & N37 $55^{\circ} 30.933^{\prime \prime}$ & E118 $34^{\prime} 33.069^{\prime \prime}$ \\
\hline Lijin County & LJ & 49 & N37²8'49.189" & E11814'06.315" \\
\hline Guangrao County & GR & 36 & N37 $17^{\circ} 17.172^{\prime \prime}$ & E118 $33^{\prime} 38.975^{\prime \prime}$ \\
\hline Dongying District & DYQ & 17 & N37 $28^{\circ} 22.546^{\prime \prime}$ & E1184ㄹ'35.488" \\
\hline
\end{tabular}

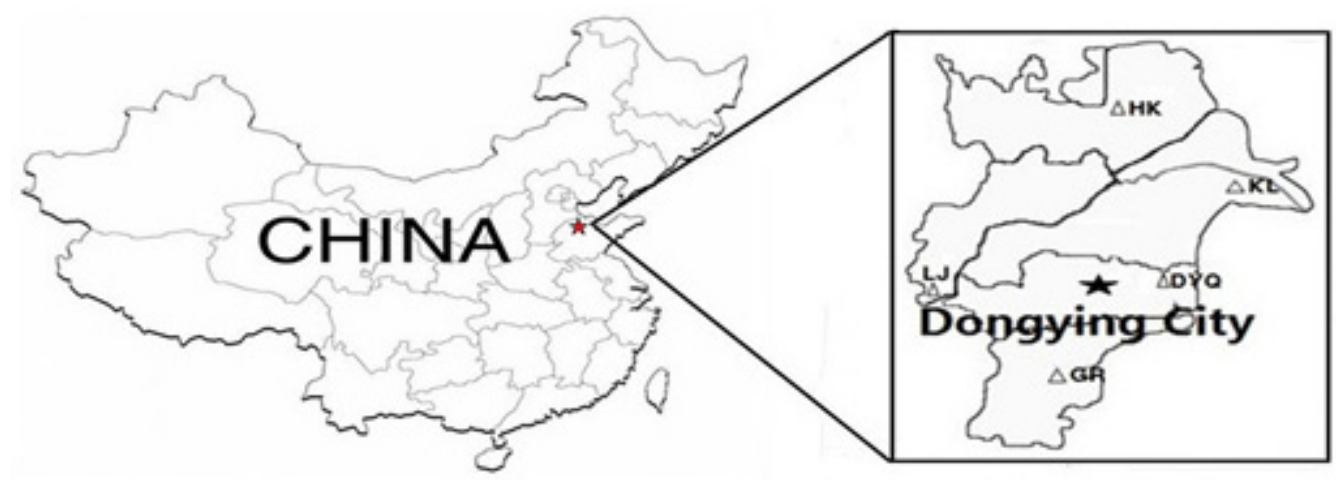

Figure 1. Location of five wild soybean populations.

\section{Seed germination and DNA extraction}

Wild soybean seeds are considered hard seeds, and they require 5-7 years to germinate under natural conditions. In this study, wild soybean seeds were soaked in concentrated sulfuric acid for 8-10 min, washed with water, and sowed in pots. Mature leaves from one plant in each accession were used for DNA extraction. The whole DNA genome was extracted with a CTAB method. 


\section{Simple sequence repeat genotyping}

Based on their position on the soybean linkage group map and the polymorphisms in cultivated soybean according to our historical record, 10 SSR markers were selected to assay the set of 195 accessions in this study (Table 2). The primer sequences with their linkage group locations are available at http://bldg6.arsusda.gov/cregan/ soymap. The $25-\mu \mathrm{L}$ PCR buffer consisted of $12.5 \mu \mathrm{L} 2 \mathrm{X}$ Taq PCR Master Mix, $1.0 \mu \mathrm{L}$ template DNA $(20 \mathrm{ng} / \mathrm{L}), 2 \mu \mathrm{L}$ $10 \mu \mathrm{M}$ forward and reverse primers, and $7.5 \mu \mathrm{L}$ autoclaved ion-exchanged water. The PCR was programmed with an initial denaturation step at $94^{\circ} \mathrm{C}$ for $5 \mathrm{~min}$, followed by 35 cycles of $94^{\circ} \mathrm{C}$ for $30 \mathrm{~s}, 45^{\circ}-55^{\circ} \mathrm{C}$ for $30 \mathrm{~s}$ and $72^{\circ} \mathrm{C}$ for $30 \mathrm{~s}$, with a final extension at $72^{\circ} \mathrm{C}$ for 10 min. Depending on the different primer combinations, three annealing temperatures $\left(45^{\circ}, 50^{\circ}\right.$, $55^{\circ} \mathrm{C}$ ) were used. PCR products were separated on $8 \%$ non-denaturing polyacrylamide gels in $1 \mathrm{X}$ TBE buffer and then visualized by silver staining.

\section{Data analysis}

The SSR bands were scored as present (1) or absent (0), each of which was treated as an independent character. Genetic diversity was conducted based on these scores. Genetic diversity was estimated according to the following parameters: 1) percentage of polymorphic loci $(\mathrm{P}), 2)$ expected heterozygosity $\left(H_{\mathrm{E}}\right)$, and 3$)$ Shannon's diversity index $(I)$. The calculation for the percentage of polymorphic loci $(\mathrm{P})$ was:

$$
\mathrm{P}=(\text { polymorphic sites/total analyzed sites) } \mathrm{x} 100 \%
$$

(Equation 1)

Expected heterozygosity $\left(H_{\mathrm{E}}\right)$ was estimated according to the formula:

$$
H_{\mathrm{E}}=1-\sum P i^{2}
$$

where $P i$ is the frequency of the $i$ th allele for the studied locus (Nei, 1987), and Shannon's diversity index $(I)$ :

$$
I=-\sum P i \ln P i
$$

where $P i$ is the frequency of the $i^{\text {th }}$ allele for the studied locus. Nei's genetic distance (D) and genetic identity $(I)$ were used to calculate the pair-wise genetic distances among the populations. All of the parameters above were calculated with the program POPGENE version 1.31 developed by Yeh et al. (1999). The Wright fixation index $\left(F_{\mathrm{ST}}\right)$ was used to estimate the variance among the populations, and the statistical significance of the index was evaluated using a 1000 times permutation of the genotypes among the populations. Gene flow $\left(N_{\mathrm{m}}\right)$, or the number of migrants entering a population in each generation, was estimated indirectly using the formula (Nei, 1973, 1978):

$$
N_{\mathrm{m}}=0.25\left(1-F_{\mathrm{ST}}\right) / F_{\mathrm{ST}}
$$

The analysis of molecular variance (AMOVA) was used to estimate the partitioning of genetic variance among and within populations. The input files for the AMOVA were 
prepared with the aid of the program DCFA1.1, written by Zhang (2001). A dendrogram was constructed with the genetic identity matrices to display population relationships using the unweighted pair-group mean algorithm (UPGMA) of NTSYSpc 2.1 (Rohlf, 2000).

\section{RESULTS}

\section{Polymorphism of SSR markers}

A total of 10 SSR markers yielded 90 bands. Of these bands, $97.78 \%$ (88 in total) were polymorphic among the 195 accessions, demonstrating that there is a high level of polymorphism at the species level. All of the primers yielded reproducible bands, but the number of amplified bands varied significantly. The number of polymorphic bands per locus ranged from five (Satt429 and Satt462) to 18 (Satt200) with an average of nine alleles per locus (Table 2). The expected heterozygosity $\left(H_{\mathrm{E}}\right)$ varied from 0.1994 (Satt586 on F) to 0.4460 (Satt571 on I), with an average of 0.3262, and Shannon's information index (I) ranged from 0.3595 (Satt429 on A2) to 0.6506 (Satt571 on I), with an average of 0.5386. All of the primers used in this research exhibited obvious polymorphism, and wild soybeans from Dongying have maintained high genetic diversity.

Table 2. Genetic richness and genetic diversity index at 10 SSR loci of wild soybean populations.

\begin{tabular}{|c|c|c|c|c|c|c|}
\hline Locus & Linkage group & Al & $H_{\mathrm{E}}$ & $I$ & $\mathrm{P}(\%)$ & $F_{\mathrm{ST}}$ \\
\hline Satt530 & $\mathrm{N}$ & 10 & 0.3202 & 0.6266 & 90 & 0.2663 \\
\hline Satt200 & A1 & 18 & 0.3483 & 0.5586 & 100 & 0.2717 \\
\hline Satt429 & A2 & 5 & 0.2069 & 0.3595 & 80 & 0.0962 \\
\hline Satt431 & $\mathrm{J}$ & 9 & 0.3334 & 0.4975 & 100 & 0.0259 \\
\hline Satt571 & I & 8 & 0.446 & 0.6506 & 100 & 0.0677 \\
\hline Satt239 & I & 8 & 0.3929 & 0.632 & 100 & 0.061 \\
\hline Satt453 & B1 & 9 & 0.3108 & 0.5079 & 100 & 0.1078 \\
\hline Satt442 & $\mathrm{H}$ & 10 & 0.4045 & 0.6157 & 100 & 0.0469 \\
\hline Satt462 & $\mathrm{L}$ & 5 & 0.2928 & 0.4256 & 100 & 0.0126 \\
\hline Satt586 & $\mathrm{F}$ & 8 & 0.1994 & 0.5117 & 100 & 0.2912 \\
\hline Mean & & 9 & 0.3262 & 0.5386 & 97.78 & 0.1533 \\
\hline
\end{tabular}

$A 1=$ total number of bands; $H_{\mathrm{E}}=$ expected heterozygosity; $I=$ Shannon's diversity index; $\mathrm{P}=$ percentage of polymorphic loci; $F_{\mathrm{ST}}=$ coefficient of gene differentiation.

\section{Genetic diversity of populations}

At the population level, the average percentage of polymorphic loci $(\mathrm{P})$ among the five populations was $84.26 \%$ (ranging from 76.60 to $89.36 \%$ ) (Table 3 ). The mean observed number of alleles $\left(N_{\mathrm{A}}\right)$ was 1.8645 with a range of 1.7826 (DYQ) to $1.9130(\mathrm{LJ})$. The effective number of alleles $\left(N_{\mathrm{E}}\right)$ showed a similar trend as the observed number of alleles $\left(N_{\mathrm{A}}\right)$ in all populations except KL. The observed number of alleles in GR and KL were the same $\left(N_{\mathrm{A}}\right.$ $=1.8667)$, but the effective number of alleles was significantly higher in $\mathrm{KL}\left(N_{\mathrm{E}}=1.6389\right)$ than in GR $\left(N_{\mathrm{E}}=1.5944\right)$, which indicated that the GR population had a higher number of extremely low frequency alleles than the KL population, and that the allelic frequencies of the KL population were comparatively well-proportioned. The mean Nei's gene diversity $\left(H_{\mathrm{E}}\right)$ and the mean Shannon's information index $(I)$ were 0.3416 and 0.5026 , respectively, demonstrating that the wild soybean populations had a relatively high level of genetic diversity. 
The average observed heterozygosity $\left(H_{\mathrm{O}}\right)$ ranged from $0.5477(\mathrm{HK})$ to $0.6689(\mathrm{GR})$, with a mean of 0.5831 , which indicated that outcrossing events occurred in all of the natural wild soybean populations.

Table 3. Analysis of genetic diversity of five wild soybean populations.
\begin{tabular}{lcccccc}
\hline Population & $N_{\mathrm{A}}$ & $N_{\mathrm{E}}$ & $H_{\mathrm{E}}$ & $H_{\mathrm{O}}$ & $I$ & $\mathrm{P}(\%)$ \\
\hline $\mathrm{LJ}$ & 1.9130 & 1.6347 & 0.3420 & 0.5678 & 0.5223 & 89.36 \\
$\mathrm{HK}$ & 1.8936 & 1.6759 & 0.3368 & 0.5477 & 0.5331 & 89.36 \\
$\mathrm{GR}$ & 1.8667 & 1.5944 & 0.3461 & 0.6689 & 0.4918 & 82.98 \\
KL & 1.8667 & 1.6389 & 0.3425 & 0.6020 & 0.5152 & 82.98 \\
$\mathrm{DYQ}$ & 1.7826 & 1.5541 & 0.3408 & 0.6233 & 0.4505 & 76.60 \\
Mean & 1.8645 & 1.6196 & 0.3416 & 0.5831 & 0.5026 & 84.26 \\
\hline
\end{tabular}

$N_{\mathrm{A}}=$ observed number of alleles; $N_{\mathrm{E}}=$ effective number of alleles; $H_{\mathrm{E}}=$ expected heterozygosity; $H_{\mathrm{O}}=$ average observed heterozygosity; $I=$ Shannon's diversity index; $\mathrm{P}=$ percentage of polymorphic loci.

\section{Genetic structure and differentiation of wild soybean in natural populations}

According to Nei's analysis of gene diversity, the genetic differentiation among the evaluated populations was $0.1533\left(F_{\mathrm{ST}}\right)$, which indicated that there was $84.67 \%$ genetic variation within the populations and that the genetic differentiation was found mainly within populations. Furthermore, the level of gene flow $\left(N_{\mathrm{m}}\right)$ was measured to be 1.3805 among populations, suggesting that there was some degree of gene exchange between the populations. Analysis of molecular variance (AMOVA) was also used to partition the SSR variation into the population components (among and within). Most of the variation (88.16\%) was detected within populations, while only a small portion of the variation $(11.84 \%)$ was attributed to variation among populations. The AMOVA test also revealed that differentiation among populations and within populations was significant $(\mathrm{P}<0.001$, Table 4$)$. The AMOVA was found to be in support of the results of the POPGENE.

Table 4. Analysis of molecular variance (AMOVA) within and among wild soybean populations.
\begin{tabular}{lcccccc}
\hline Source of variance & Sum of squares & Mean squares & Variance component & Total variance (\%) & Phist $\left(\Phi_{\text {ST }}\right)$ & P value \\
\hline Among population & 353.2395 & 88.310 & 1.9405 & 11.84 & 0.118 & $<0.001$ \\
Within population & 2744.6887 & 14.446 & 14.4457 & 88.16 & $<0.001$ \\
Total & 3097.9282 & & & & \\
\hline
\end{tabular}

\section{Phylogenetic relationship}

To further reveal the relationships among populations, cluster analyses (UPGMA) were used to generate a dendrogram based on Nei's genetic distances (Table 5). Five populations were divided into two clusters with a genetic similarity value of 0.88 (Figure 2). Cluster I consisted of three populations, which were LJ, GR, and HK, whereas cluster II contained the other two populations, which were KL and DYQ. Cluster I was again sub-divided into two, cluster i and cluster ii, with a genetic similarity value of 0.91 . Cluster ii exhibited only one population, HK, while Cluster i had two, LJ and GR. The cluster analysis indicated that there was no significant correlation between genetic distance and geographic distribution 
among populations. As shown in Table 5, the genetic distance between the populations of wild soybean ranged from 0.0733 (between DYQ and KL) to 0.1482 (between KL and GR). These results showed that there was no significant genetic differentiation among populations.

Table 5. Genetic identity (above diagonal) and genetic distance (below diagonal) between populations.

\begin{tabular}{lccccc}
\hline Population & LJ & HK & GR & KL & DYQ \\
\hline LJ & - & 0.9016 & 0.9090 & 0.8951 & 0.8738 \\
HK & 0.1036 & - & 0.9026 & 0.8976 & 0.8804 \\
GR & 0.0954 & 0.1025 & 0.1482 & - & 0.8780 \\
KL & 0.1109 & 0.1081 & 0.1301 & 0.0733 & 0.9293 \\
DYQ & 0.1350 & 0.1274 & & - \\
\hline
\end{tabular}

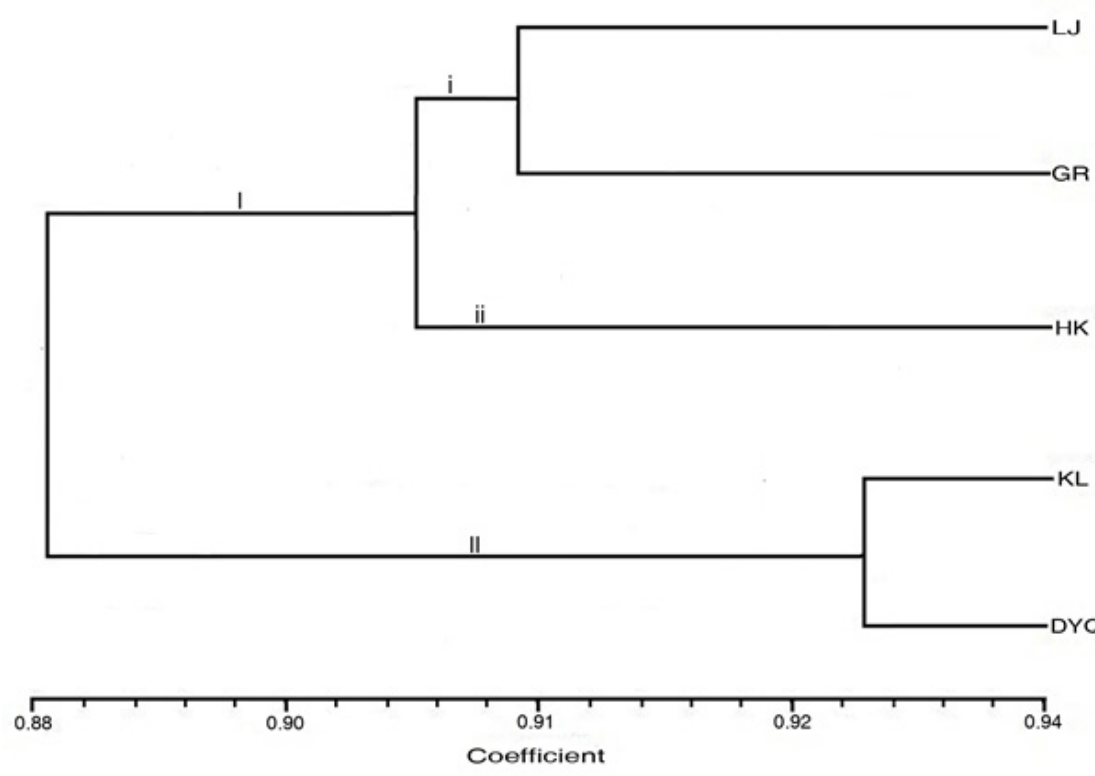

Figure 2. UPGMA dendrogram for wild soybean populations.

\section{DISCUSSION}

\section{Genetic diversity}

In this study, 90 polymorphic bands were amplified by 10 pairs of SSR primers, with an average of nine bands for each primer. Compared with the results obtained by Sun et al. (2010) (4.29 alleles using SSR primers) and Wang et al. (2014) (12.61 alleles with SSR primers), the primers in the present study appeared to be more efficient. At the species level, the percentage of polymorphic loci (P) was $97.78 \%$, the mean distribution of expected heterozygosity $\left(H_{\mathrm{E}}\right)$ was 0.3262 , and the mean distribution of Shannon's information index (I) was 0.5386 . At the population level, the average percentage of polymorphic loci (P) among the five populations was $84.26 \%$, the mean expected heterozygosity $\left(H_{\mathrm{E}}\right)$ was 
0.3416, and the mean Shannon's information index $(I)$ was 0.5026 . These results suggest that wild soybeans collected from the Dongying region of China contained abundant genetic diversity, both at the species and population levels. However, the expected heterozygosity $\left(H_{\mathrm{E}}\right)$ and Shannon's information index $(I)$ in this study were lower than those reported in previous studies (Wen et al., 2009; Xu and Li, 2011). The origin of the samples could partly account for these differences. In the present study, all of the samples were collected from limited parental materials, and are the result of inbreeding over a long period, while samples in previous studies were collected from different places. The results also implied that it is necessary to increase outcrossing to enlarge genetic diversity in the future for the conservation and management of wild soybeans.

\section{Population genetic differentiation}

Annual and selfing species allocate most of their genetic variability among populations (0.51), and relatively little within populations (Zhao, 2007). In the present study, genetic differentiation among the populations of $G$. soja was estimated to be $0.1533\left(F_{\mathrm{ST}}\right)$, which indicated that high genetic differentiation $(84.67 \%)$ existed within populations. The coefficient of gene differentiation (0.1533) was much lower than that of annual and selfing species $(0.51)$ in this study, which is consistent with the results of previous studies. Kuroda et al. (2006) indicated that, based on analysis of 77 wild soybean (Glycine soja) populations collected from Japan using 20 microsatellite primers, the $F_{\mathrm{ST}}$ was 0.192 . The results showed that the genetic differentiation among wild soybean populations was not as high as initially expected. In addition, the genetic distances in this study ranged from 0.0733 to 0.1482 , and the results showed that the degree of genetic differences was lower among the five populations and that genetic differentiation among the populations was not significant.

These results may be related to the outcrossing rates and long-distance gene flow of wild soybeans. First, in this study the mean observed heterozygosity $\left(H_{\mathrm{O}}\right)$ was 0.5831 , showing that outcrossing events occurred in the natural wild soybean populations. Although $G$. soja was previously thought to be autogamous, as is the cultivated soybean ( $G$. max), the outcrossing rate was calculated by Fujita et al. (1997) (9.3-19\%), Kuroda et al. (2006) (>10\%), and Zhao (2007) (14.9\%). The results obtained in the present study and in previous studies indicate that wild soybean has a typical mixed-mating system with a considerable proportion of inbreeding and a small proportion of outbreeding. The outcrossing rate may be related to pollination by insects. Fujita et al. (1997) found that the high outcrossing rate was supported by observations of frequent visits by honeybees and carpenter bees to flowers. Zhao (2007) also observed the movement of insects. Second, a migration rate of $N_{\mathrm{m}}=1.0$ is theoretically necessary to counter population divergence due to genetic drift, as well as to maintain genetic variations and protect inbreed-depression (Wright, 1931). In this study, the estimated gene flow based on $F_{\mathrm{ST}}$ was $1.3805(>1.0)$, indicating that it could counter strong genetic divergence among wild soybean populations to some extent. Moreover, we collected the accessions according to the findings reported by Zhao et al. (2006), and the number of individuals in each population was large, so that each population contained some identical alleles, which also leads to genetic differences among populations.

The UPGMA dendrogram obtained with our SSR data separates the five populations into two clusters. The first cluster shared LJ, GR, and HK, while KL and DYQ were assembled 
together in the second cluster. In the present study, different populations, whether their geographic distance was close or not, were clustered together, this showed that there was no recognizable correlation between the genetic diversity and the geographic distance. Similar results were reported in previous studies (Hu and Wang, 1985; Li et al., 1995), which found that no correlation existed between genetic and geographic distances even on a relatively small geographic scale. The clustering results may be attributed to the plants' similar habitats. The accessions of KL and DYQ were distributed in Dawenliu of Kenli County and the XinAn reservoir of the Dongying District. Both of the populations grew in humid environments along the rivers, and were therefore clustered together. While the accessions of LJ, GR, and HK were collected from Cai Yuan Cun of Lijin County, Wang Dao Cun of Guangrao County and a papermaking raw material factory of the Hekou District, respectively. These three populations grew in dry conditions, and were therefore clustered together.

\section{Conservation implication}

It is critical to establish the genetic diversity and variation among and within populations to choose the relevant strategy for conservation and sampling management. Our findings provide important genetic information for developing conservation strategies for $G$. soja. Considering the high level of genetic diversity and that most of the genetic variation occurs within populations, the strategy of conservation for $G$. soja should include both in situ and ex situ methods. For ex situ conservation, we need to carefully design and establish a germplasm bank for this species. We should determine the method for collecting the wild soybean germplasm according to the population genetic diversity. It is commonly believed that more than 30 individuals are needed to capture most of the genetic diversity within a population. In addition, we found that the habitat played a key role in the divergence found in this study. Hence, more attention should be paid to special circumstances with regard to the collection and preservation of wild soybean germplasm. For in situ conservation, we should pay more attention to restoring the suitable habitats and the effective population size. With the rapid development of the tourism industry, and with the exploitation of the Dongying region for road and house construction during recent years, the scale and size of G. soja populations have significantly decreased. The population system of the wild soybean has become fragmented with the fragmentation of habitats. Furthermore, it would be worthwhile to give high priority to the conservation of populations with high genetic diversity. In biological conservation, it is essential to preserve genetic diversity, which is an important source for the adaptive evolution of plant species or populations under changing environments (Wright, 1977). In addition, we found that wild soybean had a typical mixed-mating system with a great proportion of inbreeding and a small proportion of outbreeding. Increased outcrossing between individuals can promote heterozygosis, consequently enhancing genetic variation in populations or species (Charlesworth and Charlesworth, 1987); thus, with in situ conservation, we could improve the outcrossing rate of wild soybeans to facilitate the sustainable development of the species.

\section{Conflicts of interest}

The authors declare no conflict of interest. 


\section{ACKNOWLEDGMENTS}

This paper was completed with guidance from Professor Shoujin Fan and Xuejie Zhang of Shandong Normal University, China. Research supported by the Nature Science Foundation of China (\#31170173), Nature Science Foundation of Shandong Province (\#Y2008D07), and the project of Sci-tech Development Programme of Shandong Province (\#2010GSF10205, \#2014GGH210003).

\section{REFERENCES}

Abe J, Ohara M and Shimamoto Y (1992). New electrophoretic mobility variants observed in wild soybean (Glycine soja) distributed in Japan and Korea. Soybean Genet. Newsl. 19: 63-72.

Charlesworth D and Charlesworth B (1987). Inbreeding depression and its evolutionary consequences. Annu. Rev. Ecol. Syst. 18: 237-268.

Fujita R, Ohara M, Okazaki K and Shimamoto Y (1997). The extent of natural cross-pollination in wild soybean (Glycine soja). J. Hered. 88: 124-128.

Gai JY, Xu DH, Gao Z, Shimamoto Y, et al. (2000). Studies on the evolutionary relationship among eco-types of G. $\max$ and G. soja in China. Acta Agron. Sin. 26: 513-520.

Hu ZA and Wang HX (1985). Genetic structure of natural populations of wild soybean (Glycine soja) in Beijing region. Acta Bot. Sin. 27: 599-604.

Kuroda Y, Kaga A, Tomooka N and Vaughan DA (2006). Population genetic structure of Japanese wild soybean (Glycine soja) based on microsatellite variation. Mol. Ecol. 15: 959-974.

Li J, Tao Y, Zheng SZ and Zhou JL (1995). Isozymatic differentiation in local population of Glycine soja Sieb. \& Zucc. Acta Bot. Sin. 37: 669-676.

Nei M (1973). Analysis of gene diversity in subdivided populations. Proc. Natl. Acad. Sci. U. S. A. 70: 3321-3323.

Nei M (1978). Estimation of average heterozygosity and genetic distance from a small number of individuals. Genetics 89: $583-590$.

Nei M (1987). Genetic distance and molecular phylogeny. In: Population Genetics and Fishery Management (Ryman N and Utter F, eds.). The Blackburn Press, Washington, 193-223.

Rohlf FJ (2000). NTSYS-pc, numerical taxonomy and multivariate analysis system. Version 2.1. Exeter Software, Setauket, New York.

Sun XH, Liu XD, Zhao HK, Wang YM, et al. (2010). Diversity of a population of wild soybean (G. soja) growing in Longjing Conserved Region of Jilin Province. J. Jilin. Agric. Sci. 35: 1-4.

Wang D, Qiao YK, Han FX, Li GY, et al. (2010). Genetic Diversity of Glycine soja in Eastern Coastal Area of Hebei Province. Soybean Sci. 29: 555-558.

Wang KJ, Li XH and Yan MF (2014). Microsatellite markers reveal genetic diversity of wild soybean in different habitats and implications for conservation strategies (Glycine soja) in China. Conserv. Genet. 15: 605-618.

Wen ZX, Ding YL, Zhao TJ and Gai JY (2009). Genetic diversity and peculiarity of annual wild soybean (G. soja Sieb. et Zucc.) from various eco-regions in China. Theor. Appl. Genet. 119: 371-381.

Wright S (1931). Evolution in mendelian populations. Genetics 16: 97-159.

Wright S (1977). Evolution and the genetics of populations: Experimental results and evolutionary deductions v.3. University of Chicago Press, Chicago, Illinois, USA.

Wu Y, Zhao Q and Liu SC (2004). Wild soybean germplasm resource in Liaoning Province and its innovation and utilization value. Rain Fed Crop. 24: 182-183.

Xu LH and Li XH (2011). Analysis on genetic structure of wild soybeans populations by SSR markers. Soybean Sci. 30: 41-45.

Yeh FC, Yang RC and Boyle T (1999). POPGENE version 1.31 Microsoft window-based freeware for population genetic analysis. A joint project development by Francis C. Yeh and Rong-Cai Yang, University of Alberta and Tim Boyle, Centre for International Forestry Research, Bogor.

Zhang FM (2001). DCFA 1.1, a Program Companied with AMOVA to Compute the Matrix of Distance. Laboratory of Systematics and Evolutionary Botany, Institute of Botany. The Chinese Academy of Sciences, Beijing.

Zhao R (2007). Exploring basic problems in conservation genetics of wild soybean (Glycine Soja L.). Doctoral thesis, Fudan University, Shanghai. 
Zhao R, Cheng Z, Lu WF and Lu BR (2006). Estimating genetic diversity and sampling strategy for a wild soybean (Glycine soja) population based on different molecular markers. Chin. Sci. Bull. 51: 1219-1227.

Zhou XF, Zhuang BC, Wang YM and Zhao HK (2002). Population differentiation of wild soybean based on the RAPD and SSR analysis. Chin. J. Eco-Agric. 10: 6-9. 